\title{
Few-and multi-layer graphene on carbon fibers: synthesis and application
}

\begin{abstract}
In the current study, we investigated the influences of chemical vapor deposition parameters on the formation of uniform structures of few- and multi-layer graphene (FLG and MLG) as a coating phase on carbon fiber (CF). To this end, the process conditions of the chemical vapor deposition method, such as catalyst concentration, reaction temperature and time, and also carbon source flow rate, were optimized. The resulting FLG and MLG with high yields led to the modification of the CF surface by improving its properties. By applying scanning electronic microscopy, transmission electron microscopy and Raman spectroscopy, the surface morphology and structural information of the $\mathrm{G}-\mathrm{CF}$ were analyzed. It was observed that under different conditions the FLG-CF and MLG-CF were obtained with 54\%, 58\% yields and also $10.21 \mathrm{~m} 2$ $\mathrm{g}-1,8.78 \mathrm{~m} 2 \mathrm{~g}-1$ BET surface areas, respectively. Besides that, the FLG-CF and MLG-CF were used as fillers in the polypropylene (PP) composite and the effects of the number of graphene layers on the mechanical and thermal properties of the composite were analyzed. It is noteworthy to mention, composites based on the CF coated with $\mathrm{G}$ with only a few layers presented the highest surface area, strength and thermal resistance compared to those based on multi layers.
\end{abstract}

Keyword: Carbon fibers; Graphene; Microscopy; Transmission 
